\title{
Combining Highly Monochromatized EELS with CL for Probing Elementary Excitations and Their Interaction
}

Luiz Tizei ${ }^{1}$, Marcel Tencé ${ }^{1}$, Jean-Denis Blazit ${ }^{1}$, Alexandre Gloter ${ }^{2}$, Xiaoyan Li $^{1}$, Alberto Zobelli ${ }^{3}$, Laura Bocher $^{1}$, Nathalie Brun ${ }^{1}$, Marta de Frutors ${ }^{1}$, Christian Colliex ${ }^{1}$, Mathieu Kociak ${ }^{1}$ and Odile Stephan ${ }^{3}$

${ }^{1}$ CNRS, Orsay, Ile-de-France, France, ${ }^{2}$ Université Paris-Saclay, CNRS, Orsay, Ile-de-France, France, ${ }^{3}$ Université Paris-Saclay, Orsay, Ile-de-France, France

Monochromation obviously improves the energy resolution in EELS. More importantly, it provides access to a wide range of elementary excitations in the low-loss range for primary applications in condensed matter physics and in the field of nano-optics. Its association with high-brightness cold field emission sources and efficient detectors makes it possible to probe the IR-vis-UV range via spatially or momentumresolved experiments, therefore diversifying the information channels for exploring the physics of these low-energy excitations. As a matter of fact, EELS is becoming a privileged technique for probing phonons, d-d transitions, charge transfer optical gaps, excitons or plasmons, and their coupling, in complementarity to well established synchrotron-based or optical techniques. More exciting applications are emerging from the combination of such extraordinary instrumental progress with conceptual and methodological advances and complementary instrumental developments. At Orsay, some progress has been made in this direction, exploiting the combination of photon and electron beams (development of a light injection/detection system) inside the STEM. Nanometer-resolved Cathodoluminescence experiments are now available in NION Hermes microscope equipped with an electron monochromator providing a $5 \mathrm{meV}$ energy resolution in EELS spectroscopy, a low temperature side entry stage and a light collection/injection system for a combined use of CL and EELS performed both at high spectral and spatial resolution (CHROMATEM microscope).

In addition, Hanburry Brown Twiss experiments have also been developed, as derived from the quantum optics community, for detecting the emission properties of individual quantum emitters in materials and entering the field of quantum nanooptics with free electron beams.Finally, we have developed a novel way of performing stimulated electron energy-loss and energy-gain spectroscopy (sEELS/ sEEGS) experiments that does not require a pulsed gun: a regular scanning transmission electron microscope (STEM) equipped with a regular continuous electron gun has been fitted with a modified EELS detector and a light injector in the object chamber. The EELS detector has been modified so that the EELS camera can be exposed during tunable time intervals that can be synchronized with a laser hitting the sample, therefore allowing us to collect only those electrons that have interacted with the sample in presence of the laser.

Such developments are now being implemented in CHROMATEM.

Some examples will be given here on optical nanomaterials or 2D materials when exploring the intimate link between the crystal structure (including that of optical-active defects) and their optical properties.

This comprises:

- $\quad$ peculiarities in the properties of plasmonic nanowires [1] or plasmonic band gap materials with high quality factors [2] 
- $\quad$ optical properties of single photon emitters in h-BN [3] and dechalcogenides

- $\quad$ stacking effects on excitons in h-BN [4] and the appearance of new excitonic effects in dichalcogenide double layer systems

- $\quad$ strong coupling effects between plasmon and phonon modes in h-BN coupled to silver nanorods $[5]$.

first proofs of principle for sEEGS/sEELS measurements [6]

This work has received support from the National Agency for Research under the program of future investment TEMPOSCHROMATEM with the Reference No. ANR-10-EQPX-50
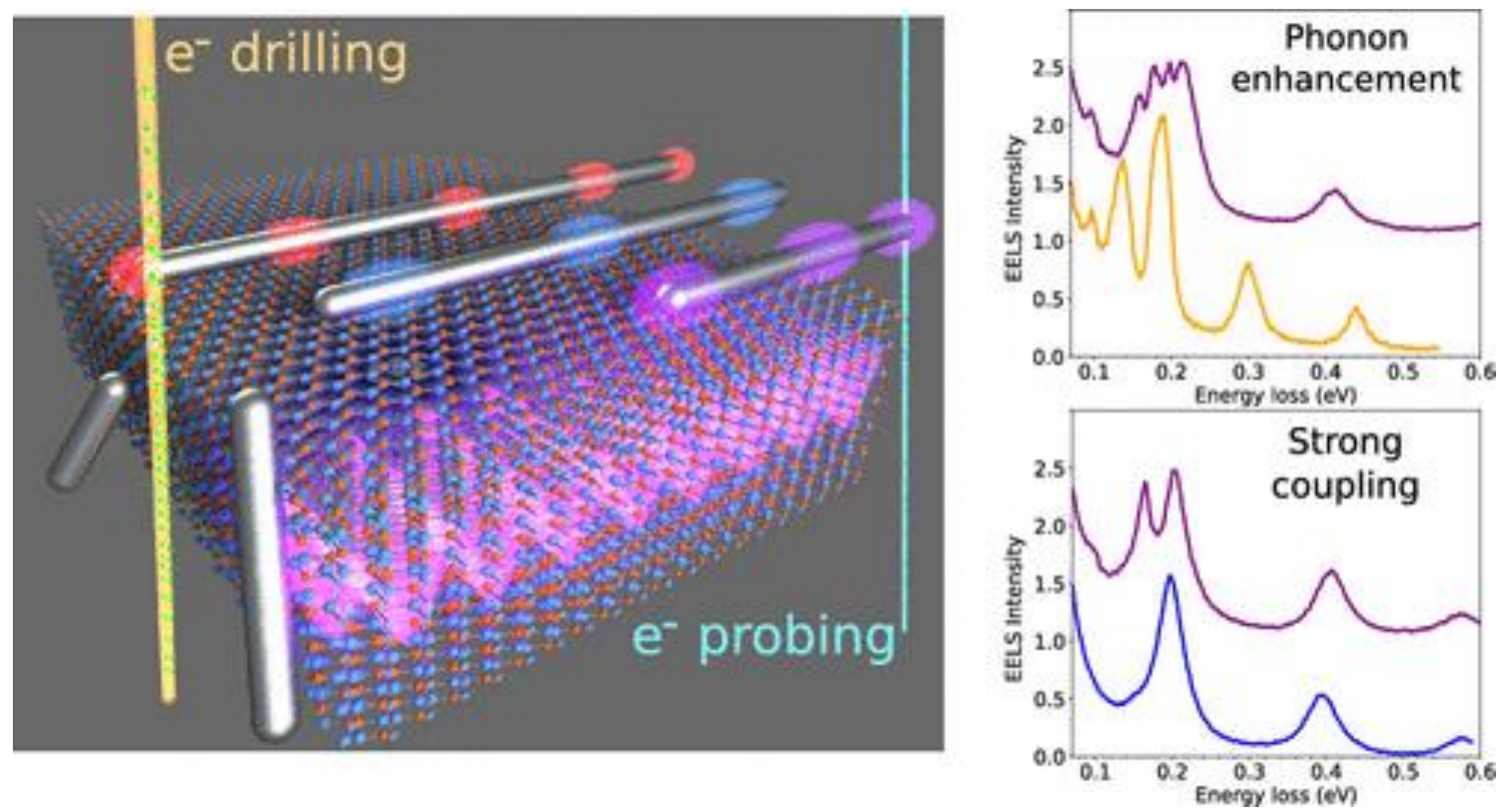

Figure 1. Coupling between h-BN optical phonons and Ag nanowires plasmonic modes. The fast electron beam in a STEM is used for first cutting the nanowires to tune the plasmon energy and second for probing by EELS the coupling spectroscopic signature when the plasmon and phonon modes are set into resonance condition. Two coupling regimes are observed: weak coupling (leading to phonon signature enhancement) and strong coupling (associated wiith phonon and plamon modes anticrossing).

\section{References}

[1] V. Mkhitaryan et al, unpublished

[2] « Emergence of point defect states in a plasmonic crystal » H. Saito et al, Phys. Rev. B 100 (2019) 245402

[3] « Bright UV single photon emission at point defects in h-BN », R. Bourrellier et al, Nano letters 16 (2016) 4317-4321

[4] « Nanometric Resolved Luminescence in h-BN Flakes: Excitons and Stacking Order » R. Bourrellier, et al, ACS Photonics 1 (2014) 857-862

[5] « Tailored Nanoscale Plasmon-Enhanced Vibrational Electron Spectroscopy ». L. Tizei et al, Nanoletters (2020), doi.org/10.1021/acs.nanolett.9b04659 
[6] «Stimulated electron energy loss and gain in an electron microscope without a pulsed electron gun », P. Das et al, Ultramicroscopy 203 (2019) 44-51 